Correction

\title{
Correction: Sugasini et al. Efficient Enrichment of Retinal DHA with Dietary Lysophosphatidylcholine-DHA: Potential Application for Retinopathies. Nutrients 2020, 12, 3114
}

\author{
Dhavamani Sugasini ${ }^{1}$, Poorna C. R. Yalagala ${ }^{1}$ and Papasani V. Subbaiah ${ }^{1,2, *(D)}$ \\ 1 Department of Medicine, Section of Endocrinology and Metabolism, University of Illinois at Chicago, \\ Chicago, IL 60612, USA; sugasini@uic.edu (D.S.); Yalagala@uic.edu (P.C.R.Y.) \\ 2 Jesse Brown VA Medical Center, Chicago, IL 60612, USA \\ * Correspondence: psubbaia@UIC.edu; Tel.: +1-312-996-8212; Fax: +1-312-413-0437
}

check for updates

Citation: Sugasini, D.; Yalagala, P.C.R.; Subbaiah, P.V. Correction: Sugasini D. et al. Efficient Enrichment of Retinal DHA with Dietary Lysophosphatidylcholine-DHA: Potential Application for Retinopathies. Nutrients 2020, 12, 3114. Nutrients 2021, 13, 2166.

https://doi.org/10.3390/nu13072166

Received: 16 April 2021

Accepted: 10 May 2021

Published: 24 June 2021

Publisher's Note: MDPI stays neutral with regard to jurisdictional claims in published maps and institutional affiliations.

Copyright: (c) 2021 by the authors. Licensee MDPI, Basel, Switzerland. This article is an open access article distributed under the terms and conditions of the Creative Commons Attribution (CC BY) license (https:// creativecommons.org/licenses/by/ $4.0 /)$.
The authors wish to make the following corrections to their recently published paper [1].

Error in Figure 2

In Figure 2 of the original article, the data for sn-1DHA LPC were repeated by mistake under sn-2 DHA LPC.

Original Figure 2.

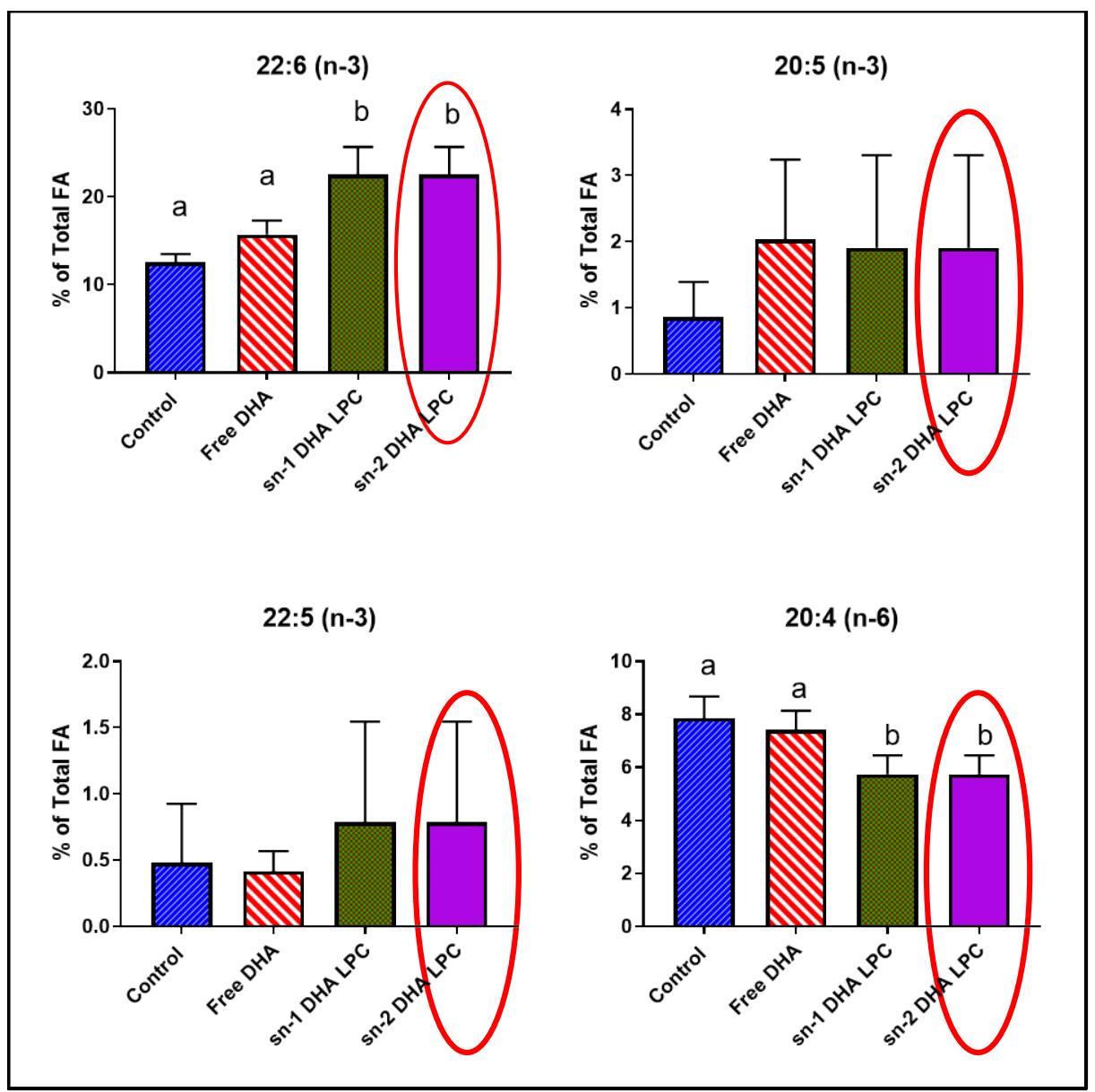

The corrected Figure 2 should be as shown below. 


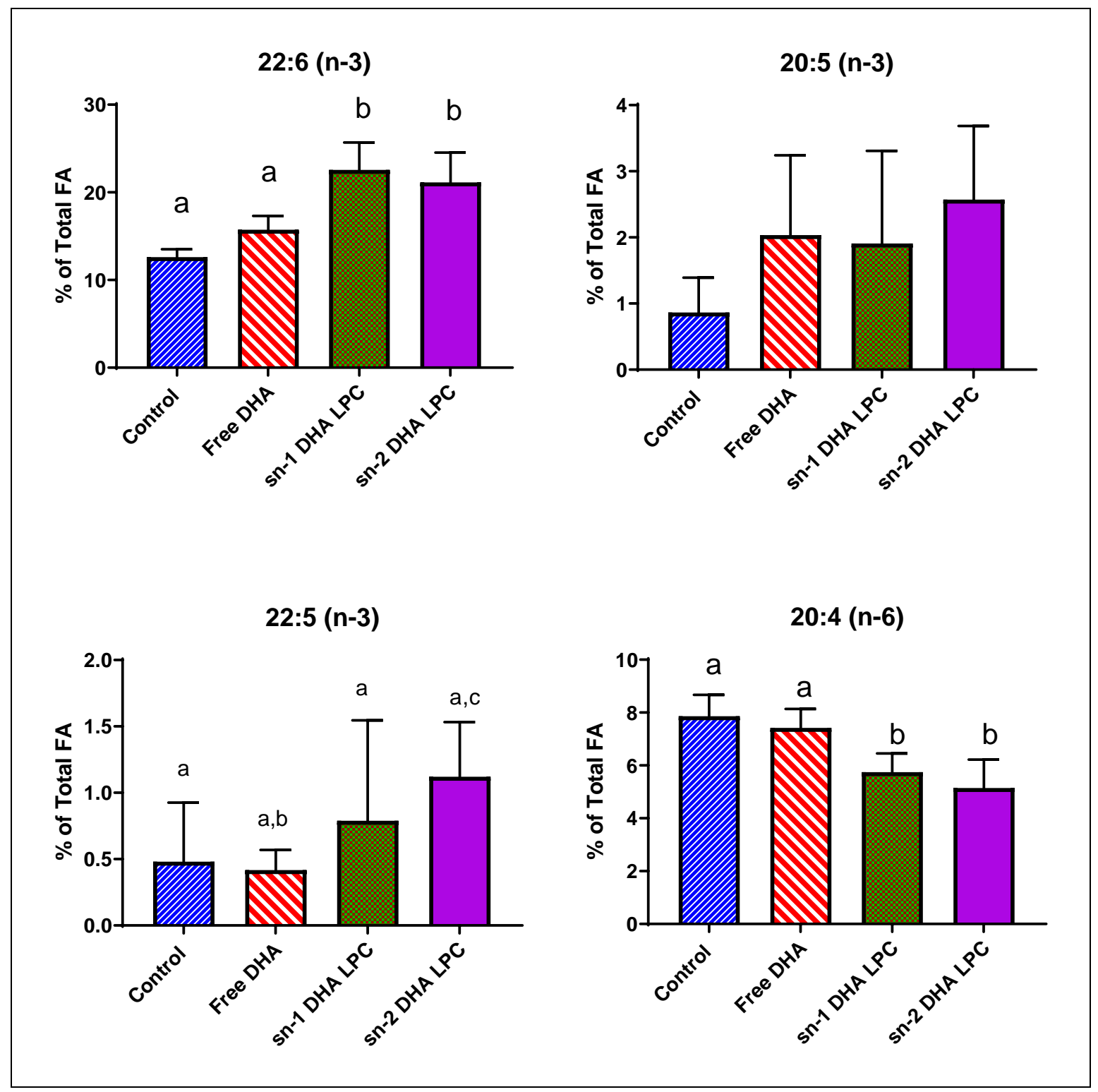

Error in Table 2

In Table 2 of the original article, the data for sn-1 DHA LPC was repeated by mistake under sn-2 DHA LPC.

Original Table 2.

Table 2. Effect of free DHA, sn-1 DHA LPC, and sn-2 DHA LPC on fatty acid composition of mouse retina.

\begin{tabular}{|c|c|c|c|c|c|c|c|c|c|c|c|c|c|c|}
\hline \multirow[b]{2}{*}{ FA } & \multicolumn{3}{|c|}{ Control } & \multicolumn{3}{|c|}{ Free DHA } & \multicolumn{3}{|c|}{ sn-1 DHA LPC } & \multicolumn{5}{|c|}{ sn-2 DHA LPC } \\
\hline & Mean & \pm & $\mathrm{SD}$ & Mean & \pm & $\mathrm{SD}$ & Mean & \pm & $\mathrm{SD}$ & & Mean & \pm & $\mathrm{SD}$ & \\
\hline $12: 0$ & 0.26 & \pm & 0.14 & 0.60 & \pm & 0.35 & 0.73 & \pm & 0.47 & & 0.73 & \pm & 0.47 & \\
\hline $14: 0$ & 0.50 & \pm & 0.25 & 0.20 & \pm & 0.17 & 0.30 & \pm & 0.17 & & 0.30 & \pm & 0.17 & \\
\hline $16: 0$ & 17.92 & \pm & 1.41 & 16.11 & \pm & 1.56 & 14.62 & \pm & 1.22 & $* *$ & 14.62 & \pm & 1.22 & $* *$ \\
\hline $16: 1$ & 0.38 & \pm & 0.17 & 0.55 & \pm & 0.28 & 0.47 & \pm & 0.34 & & 0.47 & \pm & 0.34 & \\
\hline $17: 1$ & 0.29 & \pm & 0.13 & 0.43 & \pm & 0.16 & 0.48 & \pm & 0.38 & & 0.48 & \pm & 0.38 & \\
\hline 18:0 & 18.90 & \pm & 1.05 & 17.32 & \pm & 0.66 & 15.44 & \pm & 1.50 & $* *$ & 15.44 & \pm & 1.50 & $* *$ \\
\hline $18: 1(n-9)$ & 18.23 & \pm & 0.82 & 17.14 & \pm & 0.78 & 15.56 & \pm & 1.16 & $* *$ & 15.56 & \pm & 1.16 & $* *$ \\
\hline $18: 1(n-7)$ & 4.64 & \pm & 0.48 & 4.18 & \pm & 0.34 & 3.82 & \pm & 0.62 & & 3.82 & \pm & 0.62 & \\
\hline $18: 2(n-6)$ & 0.69 & \pm & 0.45 & 0.99 & \pm & 0.65 & 1.38 & \pm & 0.49 & & 1.38 & \pm & 0.49 & \\
\hline $18: 3(n-6)$ & 0.36 & \pm & 0.19 & 0.49 & \pm & 0.36 & 0.60 & \pm & 0.47 & & 0.60 & \pm & 0.47 & \\
\hline $18: 3(n-3)$ & 0.54 & \pm & 0.43 & 0.49 & \pm & 0.15 & 0.51 & \pm & 0.42 & & 0.51 & \pm & 0.42 & \\
\hline
\end{tabular}


Table 2. Cont.

\begin{tabular}{|c|c|c|c|c|c|c|c|c|c|c|c|c|c|c|}
\hline \multirow[b]{2}{*}{$20: 0$} & \multicolumn{3}{|c|}{ Control } & \multicolumn{3}{|c|}{ Free DHA } & \multicolumn{3}{|c|}{ sn-1 DHA LPC } & \multicolumn{5}{|c|}{ sn-2 DHA LPC } \\
\hline & 1.86 & \pm & 0.69 & 1.90 & \pm & 0.64 & 1.18 & \pm & 0.72 & & 1.18 & \pm & 0.72 & \\
\hline $20: 1(n-9)$ & 3.71 & \pm & 0.42 & 3.42 & \pm & 0.59 & 2.87 & \pm & 0.60 & & 2.87 & \pm & 0.60 & \\
\hline $20: 2(n-6)$ & 1.28 & \pm & 0.84 & 0.71 & \pm & 0.50 & 0.88 & \pm & 0.95 & & 0.88 & \pm & 0.95 & \\
\hline $20: 3(n-6)$ & 0.69 & \pm & 0.54 & 1.30 & \pm & 0.66 & 1.44 & \pm & 0.35 & & 1.44 & \pm & 0.35 & \\
\hline $20: 4(n-6)$ & 7.86 & \pm & 0.81 & 7.42 & \pm & 0.72 & 5.75 & \pm & 0.71 & ** & 5.75 & \pm & 0.71 & $* *$ \\
\hline $22: 0$ & 1.20 & \pm & 0.41 & 1.08 & \pm & 0.59 & 1.24 & \pm & 0.95 & & 1.24 & \pm & 0.95 & \\
\hline $20: 5(n-3)$ & 0.86 & \pm & 0.53 & 2.03 & \pm & 1.21 & 1.91 & \pm & 1.40 & & 1.91 & \pm & 1.40 & \\
\hline $22: 2$ & 0.80 & \pm & 0.34 & 0.77 & \pm & 0.51 & 1.00 & \pm & 0.76 & & 1.00 & \pm & 0.76 & \\
\hline $22: 4(n-6)$ & 2.03 & \pm & 0.91 & 2.51 & \pm & 0.85 & 2.23 & \pm & 0.87 & & 2.23 & \pm & 0.87 & \\
\hline $22: 5(n-3)$ & 0.48 & \pm & 0.44 & 0.42 & \pm & 0.15 & 0.79 & \pm & 0.76 & & 0.79 & \pm & 0.76 & \\
\hline $22: 6(n-3)$ & 12.61 & \pm & 0.91 & 15.74 & \pm & 1.57 & 22.57 & \pm & 3.12 & $* *$ & 22.57 & \pm & 3.12 & $* *$ \\
\hline $24: 1$ & 0.78 & \pm & 0.48 & 0.51 & \pm & 0.25 & 0.49 & \pm & 0.17 & & 0.49 & \pm & 0.17 & \\
\hline 16:0 DMA & 1.16 & \pm & 0.96 & 0.75 & \pm & 0.56 & 1.02 & \pm & 0.32 & & 1.02 & \pm & 0.32 & \\
\hline 18:0 DMA & 1.25 & \pm & 1.04 & 2.10 & \pm & 0.88 & 1.96 & \pm & 0.30 & & 1.96 & \pm & 0.30 & \\
\hline 18:1 DMA & 0.73 & \pm & 0.75 & 0.85 & \pm & 0.56 & 0.78 & \pm & 0.64 & & 0.78 & \pm & 0.64 & \\
\hline
\end{tabular}

** $p<0.005$ compared to control, unpaired $t$-test adjusted with Holm-Sidak method. DMA: dimethylacetal; sn-1 and sn-2: stereospecific numbering 1 and 2 respectively; LPC: lysophosphatidylcholine; DHA: docosahexaenoic acid; F.A.: fatty acid; S.D.: standard deviation.

The corrected Table 2 should appear as below.

Table 2. Effect of free DHA, sn-1 DHA LPC, and sn-2 DHA LPC on fatty acid composition of mouse retina.

\begin{tabular}{|c|c|c|c|c|c|c|c|c|c|c|c|c|c|c|}
\hline \multicolumn{3}{|c|}{ Control } & \multicolumn{4}{|c|}{ Free DHA } & \multicolumn{3}{|c|}{ sn-1 DHA LPC } & \multicolumn{5}{|c|}{ sn-2 DHA LPC } \\
\hline FA & Mean & \pm & $\mathrm{SD}$ & Mean & \pm & SD & Mean & \pm & $\mathrm{SD}$ & & Mean & \pm & $\mathrm{SD}$ & \\
\hline $12: 0$ & 0.26 & \pm & 0.14 & 0.60 & \pm & 0.35 & 0.73 & \pm & 0.47 & & 0.98 & \pm & 0.34 & \\
\hline $14: 0$ & 0.50 & \pm & 0.25 & 0.20 & \pm & 0.17 & 0.30 & \pm & 0.17 & & 0.33 & \pm & 0.25 & \\
\hline $16: 0$ & 17.92 & \pm & 1.41 & 16.11 & \pm & 1.56 & 14.62 & \pm & 1.22 & $* *$ & 15.05 & \pm & 1.56 & $* *$ \\
\hline $16: 1$ & 0.38 & \pm & 0.17 & 0.55 & \pm & 0.28 & 0.47 & \pm & 0.34 & & 0.45 & \pm & 0.37 & \\
\hline $17: 1$ & 0.29 & \pm & 0.13 & 0.43 & \pm & 0.16 & 0.48 & \pm & 0.38 & & 0.61 & \pm & 0.47 & \\
\hline 18:0 & 18.90 & \pm & 1.05 & 17.32 & \pm & 0.66 & 15.44 & \pm & 1.50 & $* *$ & 15.40 & \pm & 0.58 & $* *$ \\
\hline $18: 1(n-9)$ & 18.23 & \pm & 0.82 & 17.14 & \pm & 0.78 & 15.56 & \pm & 1.16 & $* *$ & 15.69 & \pm & 1.10 & $* *$ \\
\hline 18:1(n-7) & 4.64 & \pm & 0.48 & 4.18 & \pm & 0.34 & 3.82 & \pm & 0.62 & & 3.87 & \pm & 0.61 & \\
\hline $18: 2(n-6)$ & 0.69 & \pm & 0.45 & 0.99 & \pm & 0.65 & 1.38 & \pm & 0.49 & & 1.05 & \pm & 0.40 & \\
\hline $18: 3(n-6)$ & 0.36 & \pm & 0.19 & 0.49 & \pm & 0.36 & 0.60 & \pm & 0.47 & & 0.28 & \pm & 0.13 & \\
\hline $18: 3(n-3)$ & 0.54 & \pm & 0.43 & 0.49 & \pm & 0.15 & 0.51 & \pm & 0.42 & & 0.49 & \pm & 0.37 & \\
\hline $20: 0$ & 1.86 & \pm & 0.69 & 1.90 & \pm & 0.64 & 1.18 & \pm & 0.72 & & 2.13 & \pm & 1.71 & \\
\hline $20: 1(n-9)$ & 3.71 & \pm & 0.42 & 3.42 & \pm & 0.59 & 2.87 & \pm & 0.60 & & 2.62 & \pm & 0.48 & \\
\hline $20: 2(n-6)$ & 1.28 & \pm & 0.84 & 0.71 & \pm & 0.50 & 0.88 & \pm & 0.95 & & 1.06 & \pm & 0.76 & \\
\hline $20: 3(n-6)$ & 0.69 & \pm & 0.54 & 1.30 & \pm & 0.66 & 1.44 & \pm & 0.35 & & 1.16 & \pm & 0.77 & \\
\hline $20: 4(n-6)$ & 7.86 & \pm & 0.81 & 7.42 & \pm & 0.72 & 5.75 & \pm & 0.71 & $* *$ & 5.14 & \pm & 1.08 & $* *$ \\
\hline $22: 0$ & 1.20 & \pm & 0.41 & 1.08 & \pm & 0.59 & 1.24 & \pm & 0.95 & & 1.27 & \pm & 1.14 & \\
\hline $20: 5(n-3)$ & 0.86 & \pm & 0.53 & 2.03 & \pm & 1.21 & 1.91 & \pm & 1.40 & & 2.57 & \pm & 1.11 & \\
\hline $22: 2$ & 0.80 & \pm & 0.34 & 0.77 & \pm & 0.51 & 1.00 & \pm & 0.76 & & 1.32 & \pm & 0.80 & \\
\hline $22: 4(n-6)$ & 2.03 & \pm & 0.91 & 2.51 & \pm & 0.85 & 2.23 & \pm & 0.87 & & 2.10 & \pm & 0.65 & \\
\hline $22: 5(n-3)$ & 0.48 & \pm & 0.44 & 0.42 & \pm & 0.15 & 0.79 & \pm & 0.76 & & 1.12 & \pm & 0.41 & \\
\hline $22: 6(n-3)$ & 12.61 & \pm & 0.91 & 15.74 & \pm & 1.57 & 22.57 & \pm & 3.12 & $* *$ & 21.14 & \pm & 3.39 & $* *$ \\
\hline $24: 1$ & 0.78 & \pm & 0.48 & 0.51 & \pm & 0.25 & 0.49 & \pm & 0.17 & & 0.54 & \pm & 0.40 & \\
\hline 16:0 DMA & 1.16 & \pm & 0.96 & 0.75 & \pm & 0.56 & 1.02 & \pm & 0.32 & & 0.98 & \pm & 0.47 & \\
\hline 18:0 DMA & 1.25 & \pm & 1.04 & 2.10 & \pm & 0.88 & 1.96 & \pm & 0.30 & & 1.78 & \pm & 1.04 & \\
\hline 18:1 DMA & 0.73 & \pm & 0.75 & 0.85 & \pm & 0.56 & 0.78 & \pm & 0.64 & & 0.89 & \pm & 0.51 & \\
\hline
\end{tabular}

** $p<0.005$ compared to control, unpaired $t$-test adjusted with Holm-Sidak method. DMA: dimethylacetal; sn-1 and sn-2: stereospecific numbering 1 and 2 respectively; LPC: lysophosphatidylcholine; DHA: docosahexaenoic acid; F.A.: fatty acid; S.D.: standard deviation.

These changes have no material impact on the conclusions of the paper. The authors would like to apologize to readers of Nutrients for this error. The published version will be updated on the article webpage, with a reference to this correction notice. 
Funding: These studies were supported by a Merit review award I01 BX004315 from US Department of Veterans Affairs (to PVS), by an equipment grant S10OD010660 from the Office of NIH Director (to PVS), and by a grant from the Alzheimer's Association, AARG-19-616614 (to DS). The content is solely the responsibility of the authors and does not necessarily represent the official views of the Veterans Administration, National Institutes of Health, or the Alzheimer's Association.

Institutional Review Board Statement: Not applicable.

Conflicts of Interest: The authors declare no conflict of interest.

\section{Reference}

1. Sugasini, D.; Yalagala, P.C.R.; Subbaiah, P.V. Efficient Enrichment of Retinal DHA with Dietary Lysophosphatidylcholine-DHA: Potential Application for Retinopathies. Nutrients 2020, 12, 3114. [CrossRef] 